\title{
ROTATIONS IN THE PRODUCT OF TWO WIENER SPACES ${ }^{1}$
}

JACOB E. BEARMAN

1. Introduction and statement of results. N. Wiener $[1]^{2}$ has defined an integral some of whose properties and applications have been investigated by R. H. Cameron and W. T. Martin [2, 3, 4]. The integral is defined over the space $C$, the space of all real-valued functions $x(t)$ which are continuous on $0 \leqq t \leqq 1$ and which satisfy the initial condition $x(0)=0$. The measure of the whole space is unity, and the measure has the properties of ordinary Lebesgue measure. The symbol $\int_{S}^{W} F(x) d_{W} x$ is used to denote the Wiener integral of a functional $F(x)$ over a Wiener measurable subset $S$ of $C$.

The present paper considers functionals of two variables, $F(x ; y)$, where $x(t) \in C$ and $y(t) \in C$, with the usual definition of the integral of $F$ over $C \otimes C$; and the behavior of this double Wiener integral is investigated when the variables are subjected to the transformation

$$
\begin{aligned}
& X(t)=\int_{0}^{t} \cos \theta(s) d x(s)-\int_{0}^{t} \sin \theta(s) d y(s), \\
& Y(t)=\int_{0}^{t} \sin \theta(s) d x(s)+\int_{0}^{t} \cos \theta(s) d y(s),
\end{aligned}
$$

where $\theta(t)$ is of bounded variation on $0 \leqq t \leqq 1$. This takes $C \otimes C$ into $C \otimes C$ in a 1-1 manner, and reduces for constant $\theta$ to

$$
\begin{aligned}
& X(t)=x(t) \cos \theta-y(t) \sin \theta, \\
& Y(t)=x(t) \sin \theta+y(t) \cos \theta,
\end{aligned}
$$

which for each $t$ is a rotation in ordinary Euclidean space. The transformations (1.1) and (1.2) shall be called "rotations" in $C \otimes C$.

The significance of the results obtained here can be appreciated only against the background of $[2,3]$ and, in particular, [4]. In view of the results obtained in those papers, it may come as a distinct surprise to find the Wiener integral behaving as intuition would suggest that an integral should behave; the results of the present paper show that under a "rotation" the Wiener integral over $C \otimes C$ of any Wiener summable functional $F(x ; y)$ is invariant, i.e., that trans-

Presented to the Society, April 26, 1947; received by the editors January 2, 1951.

${ }^{1}$ Summary of a doctoral dissertation written under R. H. Cameron at the University of Minnesota.

${ }^{2}$ Numbers in brackets refer to the bibliography at the end of the paper. 
formations of the type indicated preserve measure as well as measurability.

The final results may be stated in the following theorem.

THEOREM. Let $F(X ; Y)$ be any Wiener summable functional of $X(t)$ and $Y(t)$ on $C \otimes C$; and let $\theta(t)$ be a function of bounded variation on $0 \leqq t \leqq 1$. Then the transformation (1.1) is measure preserving and

$$
\begin{aligned}
& \int_{C}^{W} \int_{C}^{W} F[X ; Y] d_{W} X d_{W} Y \\
&=\int_{C}^{W} \int_{C}^{W} F\left[\int_{0}^{(\cdot)} \cos \theta(t) d x(t)-\int_{0}^{(\cdot)} \sin \theta(t) d y(t)\right. \\
&\left.\int_{0}^{(\cdot)} \sin \theta(t) d x(t)+\int_{0}^{(\cdot)} \cos \theta(t) d y(t)\right] d_{W} x d_{W} y .
\end{aligned}
$$

The chain of reasoning leading to this final result is in two parts, the second partly retracing the first. Part I, the angle of rotation $a$ constant, is given in $\$ \$ 2$ and 3 ; and Part II, the angle of rotation a function of $t$, is given in $\$ \S 4,5,6$. An application of the theorem is given in $\$ 7$.

2. The angle of rotation constant. Functionals restricted. Let $F(x ; y)$ be a Wiener summable functional depending on the values of $x(t)$ and $y(t)$ at only $n$ values of $t$ :

$$
F[x ; y]=f\left[x\left(t_{1}\right), x\left(t_{2}\right), \cdots, x\left(t_{n}\right) ; y\left(t_{1}\right), y\left(t_{2}\right), \cdots, y\left(t_{n}\right)\right],
$$

where $x(t) \in C$ and $y(t) \in C, 0<t_{1}<t_{2}<\cdots<t_{n} \leqq 1$, and where $f\left(u_{1}, u_{2}, \cdots, u_{n} ; v_{1}, v_{2}, \cdots, v_{n}\right) \equiv f(u ; v)$ is a Lebesgue measurable function of its $2 n$ arguments. Let the product set $S=S_{1} \otimes S_{2}$, where

$$
\begin{aligned}
& S_{1}:-\infty \leqq \xi_{j}<x\left(t_{j}\right)<\eta_{j} \leqq+\infty, \\
& S_{2}:-\infty \leqq \kappa_{j}<y\left(t_{j}\right)<\lambda_{j} \leqq+\infty,
\end{aligned} \quad j=1,2, \cdots, n,
$$

have Wiener measure given by the formula $m_{W}(S)=m_{W}\left(S_{1}\right) m_{W}\left(S_{2}\right)$, so that

$$
\begin{gathered}
m_{W}(S)=\frac{1}{\pi^{n} t_{1}\left(t_{2}-t_{1}\right) \cdots\left(t_{n}-t_{n-1}\right)} \int_{\xi_{n}}^{\eta_{n}} \cdots \int_{\xi_{1}}^{\eta_{1}} \int_{\alpha_{n}}^{\lambda_{n}} \cdots \int_{\kappa_{1}}^{\lambda_{1}} \\
\quad \exp \left[-\sum_{i=1}^{n} \frac{\left(U_{i}-U_{i-1}\right)^{2}+\left(V_{i}-V_{i-1}\right)^{2}}{t_{i}-t_{i-1}}\right] d V_{1} \cdots d V_{n} d U_{1} \cdots d U_{n},
\end{gathered}
$$

and 


$$
\begin{aligned}
& \int_{C}^{W} \int_{C}^{W} F[X ; Y] d_{W} X d_{W} Y=\frac{1}{\pi^{n} t_{1}\left(t_{2}-t_{1}\right) \cdots\left(t_{n}-t_{n-1}\right)} \\
(2.2) & \cdot \int_{-\infty}^{\infty} \stackrel{(2 n)}{\cdot} \cdot \int_{-\infty}^{\infty} f(U ; V) \exp \left[-\sum_{i=1}^{n} \frac{\left(U_{i}-U_{i-1}\right)^{2}+\left(V_{i}-V_{i-1}\right)^{2}}{t_{i}-t_{i-1}}\right] \\
& \cdot d V_{1} \cdots d V_{n} d U_{1} \cdots d U_{n},
\end{aligned}
$$

where $U_{0}=V_{0}=t_{0}=0$.

On account of an elementary property of the Wiener measure of an interval (namely, that an extra $t_{j}$ can be introduced at any point other than the given points $t_{1}, t_{2}, \cdots, t_{n}$ if the corresponding values $\xi_{j}=-\infty, \eta_{j}=+\infty$ are assigned with it; i.e., the measure is a true set function independent of the manner in which the set is described), there is no loss in generality in using the same sequence of values of $t$ for both $X$ and $Y$ in the above formulas.

Now let the transformation (1.2) hold (i.e., let $\theta(t)$ be constant), and correspondingly let

$$
\begin{aligned}
& U_{i}=u_{i} \cos \theta-v_{i} \sin \theta, \\
& V_{i}=u_{i} \sin \theta+v_{i} \cos \theta,
\end{aligned} \quad i=1,2, \cdots, n .
$$

By considering the exponent in the exponential, and the fact that the Jacobian of the transformation is unity, we see that

$$
\begin{aligned}
& \int_{-\infty}^{\infty} \stackrel{(2 n)}{\cdot} \cdot \int_{-\infty}^{\infty} f(U ; V) \\
& \cdot \exp \left[-\sum_{i=1}^{n} \frac{\left(U_{i}-U_{i-1}\right)^{2}+\left(V_{i}-V_{i-1}\right)^{2}}{t_{i}-t_{i-1}}\right] d V_{1} \cdots d V_{n} d U_{1} \cdots d U_{n} \\
& =\int_{-\infty}^{\infty} \stackrel{(2 n)}{\cdot} \int_{-\infty}^{\infty} f(u \cos \theta-v \sin \theta ; u \sin \theta+v \cos \theta) \\
& \cdot \exp \left[-\sum_{i=1}^{n} \frac{\left(u_{i}-u_{i-1}\right)^{2}+\left(v_{i}-v_{i-1}\right)^{2}}{t_{i}-t_{i-1}}\right] d v_{1} \cdots d v_{n} d u_{1} \cdots d u_{n} .
\end{aligned}
$$

Hence

$$
\begin{aligned}
\int_{C}^{W} & \int_{C}^{W} F[X ; Y] d_{W} X d_{W} Y \\
& =\int_{C}^{W} \int_{C}^{W} F[x \cos \theta-y \sin \theta ; x \sin \theta+y \cos \theta] d_{W} X d_{W} Y,
\end{aligned}
$$

which is formula (1.3) for the case $\theta=$ const. 
Thus formula (1.3) which is to be established has now been proved to hold when $\theta=$ const. and the functional depends on the values of $x$ and $y$ at only a finite number of values of $t$.

In particular, if $Q$ is an interval in $C \otimes C$, and $\mathfrak{X}_{Q}[X ; Y]$ is the characteristic functional of $Q$, it is clear that $\mathfrak{X}_{Q}[X ; Y]$ depends only on the values of $X$ and $Y$ corresponding to a finite number of values of $t$. Thus the transformation formula (2.3) holds for

$$
F[X ; Y]=\mathfrak{X}_{Q}[X ; Y] .
$$

Hence rotation through a constant angle takes intervals into measurable sets and leaves their measures invariant.

3. Angle of rotation constant. Functionals unrestricted. Since the Wiener measure is a completely additive set function, the results stated in $\$ 2$ for intervals extend directly to countable unions of intervals, and also to countable intersections of countable unions of intervals, etc. Thus, we note that for all Borel sets based on intervals in $C \otimes C$, the rotation (1.2) maps Borel sets into measurable sets which have the same measure as the original Borel sets.

Now, since (1.2) is measure preserving for Borel sets, in particular it takes null Borel sets into null sets. Thus (1.2) takes all null sets into null sets; and finally it takes measurable sets into measurable sets while leaving the measure invariant.

Since (2.3) holds for the characteristic functionals of measurable sets, it holds for simple functionals; i.e., measurable functionals taking on a finite number of different values. By monotone convergence, the result extends directly to non-negative summable functionals, and finally to unrestricted summable functionals.

4. Angle of rotation a step-function. Functionals restricted. In this section let $\theta \equiv \theta(t)$ be a step function which is left-continuous, and let $F(X ; Y)$ be restricted as in $\$ 2$. In the following discussion, take $t_{1}, t_{2}, \cdots, t_{n}$ to be any sequence of values of $t$ satisfying $0<t_{1}<t_{2}$ $<\cdots<t_{n} \leqq 1$ which include all the values of $t$ on which $x(t)$ and $y(t)$ depend and all values of $t$ where $\theta(t)$ has a finite discontinuity (this involves no essential change in the notation since any finite number of $t_{j}$ can be added at will-see the second paragraph of $\$ 2$ ).

Let $\Delta u_{i}=u_{i}-u_{i-1}$ (the use of the symbol $\Delta$ should not be taken to imply that $\Delta u_{i}$ is an increment and hence $u_{i-1}$ and $u_{i}$ are different values of the same variable; they may be independent variables), let $\Delta \sin \theta\left(t_{j}\right)=\sin \theta\left(t_{j}\right)-\sin \theta\left(t_{j-1}\right)$, with similar definitions for other variables, and let $t_{0}=u_{0}=v_{0}=0$. For each $i=1,2, \cdots, n$, let 


$$
\begin{aligned}
U_{i}= & u_{i} \cos \theta\left(t_{i}\right)-v_{i} \sin \theta\left(t_{i}\right) \\
& +\sum_{j=2}^{i}\left[v_{j-1} \Delta \sin \theta\left(t_{j}\right)-u_{j-1} \Delta \cos \theta\left(t_{j}\right)\right] \\
= & \sum_{j=1}^{i}\left[\Delta u_{j} \cos \theta\left(t_{j}\right)-\Delta v_{j} \sin \theta\left(t_{j}\right)\right]
\end{aligned}
$$

and

$$
\begin{aligned}
V_{i}= & u_{i} \sin \theta\left(t_{i}\right)+v_{i} \cos \theta\left(t_{i}\right) \\
& -\sum_{j=2}^{i}\left[u_{j-1} \Delta \sin \theta\left(t_{j}\right)+v_{j-1} \Delta \cos \theta\left(t_{j}\right)\right] \\
= & \sum_{j=1}^{i}\left[\Delta u_{j} \sin \theta\left(t_{j}\right)+\Delta v_{j} \cos \theta\left(t_{j}\right)\right] .
\end{aligned}
$$

An elementary calculation shows that the Jacobian of the transformation is unity; furthermore

$$
\sum_{i=1}^{n} \frac{\left(U_{i}-U_{i-1}\right)^{2}+\left(V_{i}-V_{i-1}\right)^{2}}{t_{i}-t_{i-1}}=\sum_{i=1}^{n} \frac{\left(u_{i}-u_{i-1}\right)^{2}+\left(v_{i}-v_{i-1}\right)^{2}}{t_{i}-t_{i-1}}
$$

and direct calculation (using (2.2) on each side) shows that

$$
\begin{aligned}
\int_{C}^{W} & \int_{C}^{W} F[X ; Y] d_{W} X d_{W} Y \\
= & \int_{C}^{W} \int_{C}^{W} f\left[\cdots, \sum_{j=1}^{i}\left\{\left[x\left(t_{j}\right)-x\left(t_{j-1}\right)\right] \cos \theta\left(t_{j}\right)\right.\right. \\
& \left.\quad-\left[y\left(t_{j}\right)-y\left(t_{j-1}\right)\right] \sin \theta\left(t_{j}\right)\right\}, \cdots ; \\
& \quad \ldots, \sum_{j=1}^{i}\left\{\left[x\left(t_{j}\right)-x\left(t_{j-1}\right)\right] \sin \theta\left(t_{j}\right)\right. \\
& \left.\left.\quad+\left[y\left(t_{j}\right)-y\left(t_{j-1}\right)\right] \cos \theta\left(t_{j}\right)\right\}, \cdots\right] d_{W} x d_{W} y .
\end{aligned}
$$

For $t_{j-1}<t \leqq t_{j}$, we have $\theta(t)=\theta\left(t_{j}\right)$ since $\theta(t)$ is a left-continuous step-function having no discontinuities except possibly at $t_{1}, t_{2}, \cdots$, $t_{n}$. Now

$$
\begin{aligned}
\sum_{j=1}^{i}\left\{\left[x\left(t_{j}\right)-x\left(t_{j-1}\right)\right] \cos \theta\left(t_{j}\right)\right. & \left.-\left[y\left(t_{j}\right)-y\left(t_{j-1}\right)\right] \sin \theta\left(t_{j}\right)\right\} \\
& =\int_{0}^{t_{i}} \cos \theta(t) d x(t)-\int_{0}^{t_{i}} \sin \theta(t) d y(t),
\end{aligned}
$$


and the same equation holds with $\cos$ replaced by $\sin$ and $\sin$ by - cos. Thus $(1.3)$ is valid for $F(X ; Y)$ which depends only on the values of $X$ and $Y$ at a finite number of values of $t$ and for $\theta(t)$ a left-continuous step-function.

5. Angle of rotation variable. Functional restricted. Let $\theta(t)$ be a left-continuous function of bounded variation, and let $F(X ; Y)$ be restricted as in $\$ 2$. Further, since a limiting argument must be used to generalize $\theta$ from a step-function to a function of bounded variation, we require that the function $f$, in terms of which $F(X ; Y)$ is defined, be bounded and continuous in all its arguments over its whole range of definition.

Let $\left\{t_{i}^{*}\right\}, i=1,2, \cdots, \nu$, be a set of values of $t$ such that $F(X ; Y)$ depends only on $X\left(t_{i}^{*}\right)$ and $Y\left(t_{i}^{*}\right), i=1,2, \cdots, \nu$. Form a partition of $[0,1]$ into $m$ equal parts; let the number of distinct values of $t$ in $\left\{t_{i}^{*}\right\}$ and the above partition be $n$. Let these distinct values be $\left\{t_{j}\right\}, j=1,2, \cdots, n$. Let $n_{i}$ be the subscript of $t_{i}^{*}$ in the new notation, i.e., $t_{j}=t_{i}^{*}$ if $j=n_{i}$. Certainly $m \leqq n \leqq m+\nu$. Form the stepfunction $\theta_{n}(t)=\theta\left(t_{j}\right)$ on $t_{j-1}<t \leqq t_{j}$. For fixed $\nu$, it is clear that $n$ and all $t_{j}$ and all $n_{i}$ depend on $m$, while $\theta_{n}(t)$ also really depends on $m$. Note in particular that $m \rightarrow \infty$ and $n \rightarrow \infty$ are equivalent, so that the use of the latter rather than the former causes no ambiguity; and the dependence of the step-function, etc., on $m$ will not be indicated, in the interest of avoiding additional notational difficulties. By the results of $\$ 4$ we have

$$
\begin{aligned}
\int_{C}^{W} \int_{C}^{W} F[X ; Y] d_{W} X d_{W} Y=\int_{C}^{W} \int_{C}^{W} F\left[\int_{0}^{(\cdot)} \cos \theta_{n}(t) d x(t)\right. \\
-\int_{0}^{(\cdot)} \sin \theta_{n}(t) d y(t) ; \int_{0}^{(\cdot)} \sin \theta_{n}(t) d x(t) \\
\left.+\int_{0}^{(\cdot)} \cos \theta_{n}(t) d y(t)\right] d_{W} x d_{W} y .
\end{aligned}
$$

Consider any one of the four Stieltjes integrals in (5.1), the results of all four integrals going through similarly; e.g., consider

$$
\int_{0}^{t_{i}^{*}} \cos \theta_{n}(s) d x(s)=\sum_{j=1}^{n_{i}} \cos \theta\left(t_{j}\right)\left[x\left(t_{j}\right)-x\left(t_{j-1}\right)\right] .
$$

Now the integrals $\int_{0}^{t} \cos \theta(s) d x(s)$, etc., exist for $0 \leqq t \leqq 1$ since $\theta(s)$ and hence $\cos \theta(s)$ and $\sin \theta(s)$ are of bounded variation on $[0, t]$ and $x(s)$ is continuous on this interval. Hence in particular they exist 
if $t=t_{i}^{*}, i=1,2, \cdots, \nu$. Thus by the definition of a RiemannStieltjes integral,

$$
\begin{aligned}
\int_{0}^{t_{i}^{*}} \cos \theta(s) d x(s) & =\lim _{n \rightarrow \infty} \sum_{j=1}^{n_{i}} \cos \theta\left(t_{j}\right)\left[x\left(t_{j}\right)-x\left(t_{j-1}\right)\right] \\
& =\lim _{n \rightarrow \infty} \int_{0}^{t_{i}^{*}} \cos \theta_{n}(s) d x(s), \quad i=1,2, \cdots, \nu ;
\end{aligned}
$$

and the same is true when cos is replaced by $\sin$ or $x$ by $y$ or both.

Let $X$ and $Y$ be defined in terms of $x$ and $y$ by (1.1), and similarly let

$$
\begin{aligned}
& X_{n}(t)=\int_{0}^{t} \cos \theta_{n}(s) d x(s)-\int_{0}^{t} \sin \theta_{n}(s) d y(s), \\
& Y_{n}(t)=\int_{0}^{t} \sin \theta_{n}(s) d x(s)+\int_{0}^{t} \cos \theta_{n}(s) d y(s) .
\end{aligned}
$$

Then by (5.2), etc., we have for $i=1,2, \cdots, \nu$,

$$
\lim _{n \rightarrow \infty} X_{n}\left(t_{i}^{*}\right)=X\left(t_{i}^{*}\right) \text { and } \lim _{n \rightarrow \infty} Y_{n}\left(t_{i}^{*}\right)=Y\left(t_{i}^{*}\right) .
$$

But by the assumptions of this section, $F(X ; Y)$ depends only on the values of $X$ and $Y$ at $t=t_{i}^{*}(i=1,2, \cdots, \nu)$, and it is a continuous function of those values. Hence

$$
\lim _{n \rightarrow \infty} F\left(X_{n} ; Y_{n}\right)=F(X ; Y) .
$$

Then, since by the assumptions of this section $F$ is bounded, it follows from (5.1), (5.3), (5.4), (1.1), and the principle of bounded convergence that (1.3) holds. We have now established (1.3) under the assumptions that $\theta(t)$ is a left-continuous function of bounded variation and $F(X ; Y)=f\left[x\left(t_{1}^{*}\right), \cdots, x\left(t_{v}^{*}\right) ; y\left(t_{1}^{*}\right), \cdots, y\left(t_{v}^{*}\right)\right]$, where $f$ is bounded and continuous.

6. The general case. We now remove the assumptions of the last section. We note first that any function of bounded variation can be made left-continuous by changing its values at its countable set of discontinuities and that these changes leave $\int_{0}^{t} \cos \theta(s) d x(s)$, etc., invariant for all values of $t$ on $[0,1]$. Hence if $(1.3)$ holds when $\theta(t)$ is normalized to be left-continuous, it holds for the original $\theta(t)$; and the assumption that $\theta(t)$ be left-continuous can therefore be dropped. 
Next we note that (1.3) holds if $F[X ; Y]$ is the characteristic functional of an interval and $\theta(t)$ is any function of bounded variation. In this case all the assumptions of the last section are satisfied except for the requirement that the function $f$ on which $F$ depends be continuous. But $f$ is now the characteristic function of an interval in $2 \nu$ dimensions, and can be approximated arbitrarily closely in the pointwise sense by $2 \nu$-dimensional continuous trapezoidal functions which are uniformly bounded. Since (1.3) holds for $F$ defined in terms of these functions, it holds for the original $F$ by bounded convergence. This brings us to the same point in the argument as was reached (for constant $\theta$ ) at the end of $\$ 2$; and from this point on the argument proceeds to the general result in a manner strictly analogous to that of $\$ 3$. Thus the theorem is proved. We note in particular that when $\theta(t)$ is of bounded variation, (1.1) leaves measurability and measure invariant.

7. An application. Aside from the theoretical importance of the results presented here and their use in extending the general theory of the Wiener integral [5], the formulae given here have the immediate utility of allowing the evaluation of Wiener integrals previously unknown. As an illustration, let us consider the integral

$$
A \equiv \int_{C}^{W} \int_{C}^{W} \exp \left[2 \lambda^{2} \int_{0}^{1} x(t) y(t) d t\right] d_{W} x d_{W} y .
$$

Applying to $A$ the transformation formula (2.3), with $\theta=\pi / 4$, we obtain

$$
A=B \equiv \int_{C}^{W} \int_{C}^{W} \exp \left\{\lambda^{2} \int_{0}^{1}\left[x^{2}(t)-y^{2}(t)\right] d t\right\} d_{W} x d_{W} y .
$$

The summability of the integrand of $B$ and hence of $A$ can be seen for appropriate values of $\lambda$ by applying the Fubini theorem to $B$ and evaluating the resulting single Wiener integrals by equation (13.12) of [3]; thus when $-\pi / 2<\lambda<\pi / 2$,

$$
\begin{aligned}
B & =\int_{C}^{W} \exp \left[\lambda^{2} \int_{0}^{1} x^{2}(t) d t\right] d_{W} x \cdot \int_{C}^{W} \exp \left[-\lambda^{2} \int_{0}^{1} y^{2}(t)\right] d_{W} y \\
& =[\sec \lambda \operatorname{sech} \lambda]^{1 / 2}
\end{aligned}
$$

and the summability of the two factor integrals establishes the summability of $B$.

Now suppose we reduce $A$ to a single Wiener integral by the earlier theory. Let $\alpha(t)=\int_{1}^{t} y(s) d s$ and we have by Fubini's theorem and 
equation (7.5) of [2],

$$
\begin{aligned}
A & =\int_{C}^{W}\left\{\int_{C}^{W} \exp \left[2 \lambda^{2} \int_{0}^{1} x(t) d \alpha(t)\right] d_{W} x\right\} d_{W} y \\
& =\int_{C}^{W}\left\{\exp \left[\lambda^{4} \int_{0}^{1} \alpha^{2}(t) d t\right]\right\} d_{W} y \\
& =\int_{C}^{W} \exp \left[\lambda^{4} \int_{0}^{1}\left\{\int_{1}^{t} y(s) d s\right\}^{2} d t\right] d_{W} y .
\end{aligned}
$$

Setting this equal to $B$, we obtain the new formula

$$
\int_{C}^{W} \exp \left[\lambda^{4} \int_{0}^{1}\left\{\int_{1}^{t} y(s) d s\right\}^{2} d t\right] d_{W} y=[\sec \lambda \operatorname{sech} \lambda]^{1 / 2}
$$

This formula has been shown to be valid for $-\pi / 2<\lambda<\pi / 2$, but its validity could be extended by the theory of analytic continuation to all complex values for which the integral converges; i.e., for all $\lambda$ such that $\operatorname{Re}\left(\lambda^{4}\right)<\pi^{4} / 16$.

\section{BIBLIOGRAPHY}

1. N. Wiener, Generalized harmonic analysis, Acta Math. vol. 55 (1930) pp. 117258; especially pp. 214-234.

2. R. H. Cameron and W. T. Martin, Transformations of Wiener integrals under translations, Ann. of Math. vol. 45 (1944) pp. 386-396.

3. - Transformations of Wiener integrals under a general class of linear transformations, Trans. Amer. Math. Soc. vol. 58 (1945) pp. 184-219.

4. - The behavior of measure and measurability under change of scale in Wiener space, Bull. Amer. Math. Soc. vol. 53 (1947) pp. 130-137.

5. H. A. Colson, An existence theorem for a generalized Riemann-Stieltjes integral, Doctoral dissertation, University of Minnesota, November, 1949.

The University of Minnesota 